\title{
Stochastic Resonance in a Multistable System Driven by Gaussian Noise
}

\author{
Peiming Shi, ${ }^{1}$ Pei Li, ${ }^{1}$ Shujun An, ${ }^{1}$ and Dongying Han ${ }^{2}$ \\ ${ }^{1}$ College of Electrical Engineering, Yanshan University, Qinhuangdao 066004, China \\ ${ }^{2}$ College of Vehicles and Energy, Yanshan University, Qinhuangdao 066004, China \\ Correspondence should be addressed to Peiming Shi; peiming.shi@163.com
}

Received 1 December 2015; Revised 23 January 2016; Accepted 4 February 2016

Academic Editor: Taher S. Hassan

Copyright (c) 2016 Peiming Shi et al. This is an open access article distributed under the Creative Commons Attribution License, which permits unrestricted use, distribution, and reproduction in any medium, provided the original work is properly cited.

Stochastic resonance (SR) is investigated in a multistable system driven by Gaussian white noise. Using adiabatic elimination theory and three-state theory, the signal-to-noise ratio (SNR) is derived. We find the effects of the noise intensity and the resonance system parameters $b, c$, and $d$ on the SNR; the results show that SNR is a nonmonotonic function of the noise intensity; therefore, a multistable SR is found in this system, and the value of the peak changes with changing the system parameters.

\section{Introduction}

Stochastic resonance (SR) is first introduced by Benzi et al. [1] in 1981. In the past decades, SR has received considerable attention in the field of meteorology, and the topic has flourished in physics and neuroscience and weak signal detection [2-6].

There have been many theoretical developments of SR in conventional bistable systems [7-12]. Recently, there have appeared some extensions of SR, such as stochastic resonance in a harmonic oscillator [13], ghost stochastic resonance in the FitzHugh-Nagumo neuron model $[14,15]$, Transition in a Bistable Duffing System [16], time delay SR [17], trichotomous noise induced SR in a linear system [18], and superthreshold SR [19]. Literature [20-22] proposes a new model of multistable system. However, [7-22] did not study the SNR. In this paper, we use the model of multistable system driven by periodic signal and white noise which can realize the maximum utilization of noise and obtain better detection effects. So it is necessary to discuss the SNR of the multistable system.

In order to describe SR, McNamara and Wiesenfeld [7] introduced the signal-to-noise ratio, which is often used as an indicator of signal processing performance. Numerous studies have been developed to explain SR in continuous time using tools of statistical physics.
Literature [25] studied a solution of Kramers turnover problem for the case of two symmetric deep wells connected through a single shallow well; literature [26] analysed the occurrence of vibrational resonance in a damped quantic oscillator with double-well and triple-well potentials driven by both low-frequency force and high-frequency force; the splitting of the Kramers escape rate in an overdamped system with a triple-well potential was studied in [27].

The paper is organized as follows. In Section 2, we present the model for the multistable system. Then, the expression of the signal-to-noise ratio is derived. In Section 3, the effects of noise intensity and the resonance system parameters $b, c$, and $d$ on SNR are discussed. A discussion of the effects concludes the paper in Section 4.

\section{SNR of Multistable SR}

The model of multistable SR is a multistable nonlinear system driven by periodic signal and white noise. The equation can be written as follows:

$$
\frac{d x}{d t}=-\frac{d U(x)}{d x}+s(t)+\eta(t)
$$

where $s(t)=A \cos (2 \pi f t)$ is the input signal, $A$ is the periodic signal amplitude, $f$ is the driving frequency, $\eta(t)=$ $\sqrt{2 D} \varepsilon(t)$ in which $D$ is the noise intensity, and $\varepsilon(t)$ represents 


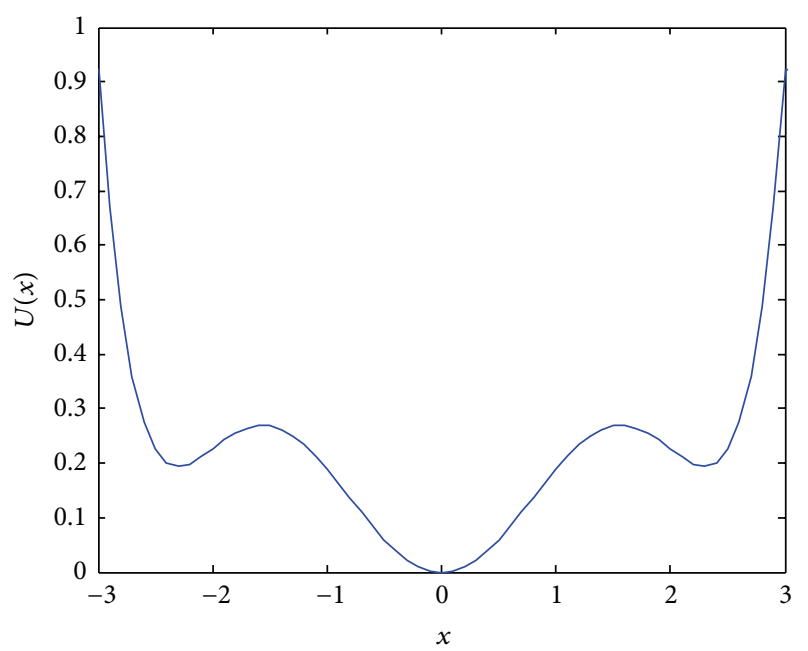

Figure 1: The multistable potential function $U(x)$.

a Gaussian white noise with zero mean and unit variance. $x(t)$ is the multistable SR output signal. The potential function for the above multistable system can be denoted as $[21,22]$

$$
U(x)=\frac{b}{2} x^{2}+\frac{c}{4} x^{4}+\frac{d}{6} x^{6}
$$

where $b, c$, and $d$ are system parameters. As shown in Figure 1, the potential function $U(x)$ is symmetrical and has three stable points $\left(-x_{2}, x_{0}\right.$ and $\left.x_{2}\right)$ and two unstable points $\left(-x_{1}, x_{1}\right)$ :

$$
\begin{aligned}
& x_{0}=0, \\
& x_{1}=\sqrt{\frac{-1}{2 d}\left(c+\sqrt{c^{2}-4 b d}\right)}, \\
& x_{2}=\sqrt{\frac{-1}{2 d}\left(c-\sqrt{c^{2}-4 b d}\right)} .
\end{aligned}
$$
by

From (1) and (2), the Fokker-Planck equation [26] is given

$$
\begin{aligned}
& \frac{\partial \rho(x, t)}{\partial t} \\
& =-\frac{\partial}{\partial x}\left[-b x-c x^{3}-d x^{5}+A \cos (2 \pi f t) \rho(x, t)\right] \\
& \quad+D \frac{\partial^{2}}{\partial x^{2}} \rho(x, t) .
\end{aligned}
$$

Formula (4) contains nonlinear components, so it cannot obtain the steady state solution.

When the input signal and noise intensity are very small,

$$
\begin{aligned}
& A \ll 1, \\
& D \ll 1 .
\end{aligned}
$$

The whole $x$ area can be divided into three attraction domains; the first is the attraction domain of the steady-state solution $x=-\sqrt{(-1 / 2 d)\left(c-\sqrt{c^{2}-4 b d}\right)}$, the second is the attraction domain of the steady-state solution $x=0$, and the last is the attraction domain of the steady-state solution $x=$ $\sqrt{(-1 / 2 d)\left(c-\sqrt{c^{2}-4 b d}\right)}$. In the three attraction domains, the total probability of them contains, respectively [20],

$$
\begin{aligned}
& P_{1}(t)=\int_{-\infty}^{-x_{1}} \rho(x, t) d x, \\
& P_{2}(t)=\int_{-x_{1}}^{x_{1}} \rho(x, t) d x, \\
& P_{3}(t)=\int_{x_{1}}^{+\infty} \rho(x, t) d x .
\end{aligned}
$$

Obviously, $P_{1}(t)+P_{2}(t)+P_{3}(t)=1$, when the frequency of input signal is very low

$$
f \ll 1 .
$$

In the condition of adiabatic approximation, we can get the master equation for the probability of exchange among the three quantities by simplifying (3):

$$
\begin{aligned}
P_{1}^{\prime}(t) & =-R_{1}(t) P_{1}(t)+\frac{1}{2} R_{2}(t) P_{2}(t) \\
& =\frac{1}{2} R_{2}(t)-\left[R_{1}(t)+R_{2}(t)\right] P_{1}(t), \\
P_{2}^{\prime}(t) & =-R_{2}(t) P_{2}(t)+R_{1}(t) P_{1}(t)+R_{3}(t) P_{3}(t), \\
P_{3}^{\prime}(t) & =-R_{3}(t) P_{3}(t)+\frac{1}{2} R_{2}(t) P_{2}(t),
\end{aligned}
$$

where $R_{1,2,3}(t)$ are the escape rate [7]. They are considered as function of a weak periodic signal $A \cos (2 \pi f t)$, when $A \ll 1$, under the adiabatic approximation, the escape rate of $R_{1,2,3}(t)$ series expansion, ignoring the higher order terms, you can get the following expression:

$$
\begin{aligned}
& R_{1}=R_{3} \\
& \quad=\frac{1}{2}\left(R_{0}+R_{1} \beta \cos (2 \pi f t)+R_{2} \beta^{2} \cos ^{2}(2 \pi f t)+\cdots\right), \\
& R_{2} \\
& \quad=\frac{1}{2}\left(R_{0}-R_{1} \beta \cos (2 \pi f t)+R_{2} \beta^{2} \cos ^{2}(2 \pi f t)-\cdots\right) ;
\end{aligned}
$$

then,

$$
R_{1}+R_{2}=R_{0}+R_{2} \beta^{2} \cos ^{2}(2 \pi f t) .
$$

Equations (8) can be solved as

$$
\begin{aligned}
& P_{1}(t)=\frac{1}{4}\left\{e^{-R_{0}\left(t-t_{0}\right)}\left[2 P_{1}\left(t_{0}\right)-1+m\right]+1-n\right\}, \\
& P_{2}(t)=\frac{1}{2}\left\{e^{-R_{0}\left(t-t_{0}\right)}\left[2 P_{2}\left(t_{0}\right)-1-m\right]+1+n\right\}, \\
& P_{3}(t)=\frac{1}{4}\left\{e^{-R_{0}\left(t-t_{0}\right)}\left[2 P_{3}\left(t_{0}\right)-1+m\right]+1-n\right\},
\end{aligned}
$$


where

$$
\begin{array}{r}
m=\frac{R_{1} \beta \cos \left(2 \pi f t_{0}-\theta\right)}{\left(R_{0}^{2}+(2 \pi f)^{2}\right)^{1 / 2}}, \\
n=\frac{R_{1} \beta \cos (2 \pi f t-\theta)}{\left(R_{0}^{2}+(2 \pi f)^{2}\right)^{1 / 2}}, \\
\sin \theta=\frac{2 \pi f}{\left(R_{0}^{2}+(2 \pi f)^{2}\right)^{1 / 2}}, \\
\cos \theta=\frac{R_{0}}{\left(R_{0}^{2}+(2 \pi f)^{2}\right)^{1 / 2}} .
\end{array}
$$

When $t_{0} \rightarrow-\infty, P_{1,2,3}(t)$ approaches $P_{1,2,3}^{s}(t)$ :

$$
\begin{aligned}
& P_{1}^{s}(t)=P_{3}^{s}(t)=\frac{1}{4}(1-n), \\
& P_{2}^{s}(t)=\frac{1}{2}(1+n) .
\end{aligned}
$$

Let $P_{i}(t+\tau \mid j, t)$ donate the probability to the system which is in $j$ area at $t$ moment when it is in $i$ area at $t+\tau$ moment $(i, j=1,2,3)$ :

$$
\begin{aligned}
& P_{1}(t+\tau \mid 1, t)=\frac{1}{4}\left[e^{-R_{0} \tau}(-1+n)+1-q\right], \\
& P_{1}(t+\tau \mid 2, t)=\frac{1}{4}\left[e^{-R_{0} \tau}(1+n)+1-q\right], \\
& P_{2}(t+\tau \mid 2, t)=\frac{1}{2}\left[e^{-R_{0} \tau}(1-n)+1+q\right], \\
& P_{2}(t+\tau \mid 1, t)=\frac{1}{4}\left[e^{-R_{0} \tau}(-1-n)+1+q\right], \\
& P_{2}(t+\tau \mid 3, t)=\frac{1}{4}\left[e^{-R_{0} \tau}(-1-n)+1+q\right], \\
& P_{3}(t+\tau \mid 2, t)=\frac{1}{4}\left[e^{-R_{0} \tau}(1+n)+1-q\right], \\
& P_{3}(t+\tau \mid 3, t)=\frac{1}{4}\left[e^{-R_{0} \tau}(-1+n)+1-q\right] .
\end{aligned}
$$

In the progressive state, the correlation function of random variable is given by

$$
\begin{aligned}
& \langle x(t) x(t+\tau)\rangle=\lim _{t_{0} \rightarrow-\infty} \iint x y P_{y}(t+\tau \mid x, t) \rho(x, t) \\
& =\left(-x_{2}\right)\left(-x_{2}\right) P_{1}(t+\tau \mid 1, t) P_{1}^{s}(t) \\
& \quad+\left(-x_{2}\right) x_{0} P_{2}(t+\tau \mid 1, t) P_{1}^{s}(t)
\end{aligned}
$$

$$
\begin{aligned}
& +x_{0} x_{0} P_{2}(t+\tau \mid 2, t) P_{2}^{s}(t) \\
& +x_{0}\left(-x_{2}\right) P_{1}(t+\tau \mid 2, t) P_{2}^{s}(t) \\
& +x_{0} x_{2} P_{3}(t+\tau \mid 2, t) P_{2}^{s}(t) \\
& +x_{2} x_{2} P_{3}(t+\tau \mid 3, t) P_{3}^{s}(t) \\
& +x_{2} x_{0} P_{2}(t+\tau \mid 3, t) P_{3}^{s}(t) \\
& =\frac{1}{8} x_{2}^{2}\left[e^{-R_{0}|\tau|}\left(-1+2 n-n^{2}\right)+1-q-n+n q\right] \\
& +\frac{1}{4} x_{0}^{2}\left[e^{-R_{0}|\tau|}\left(1-n^{2}\right)+1+q+n+n q\right] .
\end{aligned}
$$

The correlation function is not only related with the time interval but also related with the start value of the time. So we take the average value of the correlation function

$$
\begin{aligned}
\langle x & (t) x(t+\tau)\rangle_{\text {Average }}=f \int_{0}^{1 / f}\langle x(t) x(t+\tau)\rangle d t \\
= & \left(\frac{-c}{8 d}-\frac{1}{4} \sqrt{\frac{b}{d}}\right) \\
\cdot & \left\{e^{-R_{0}|\tau|}\left[-1-\frac{R_{1}^{2} \beta^{2}}{2\left(R_{0}^{2}+(2 \pi f)^{2}\right)}\right]+1\right. \\
+ & \left.\frac{R_{1}^{2} \beta^{2} \cos (2 \pi f \tau)}{2\left(R_{0}^{2}+(2 \pi f)^{2}\right)}\right\}+\left[\frac{-1}{8 d}\left(c+\sqrt{c^{2}-4 b d}\right)\right] \\
\cdot & \left\{e^{-R_{0}|\tau|}\left[1-\frac{R_{1}^{2} \beta^{2}}{2\left(R_{0}^{2}+(2 \pi f)^{2}\right)}\right]+1\right. \\
+ & \left.\frac{R_{1}^{2} \beta^{2} \cos (2 \pi f \tau)}{2\left(R_{0}^{2}+(2 \pi f)^{2}\right)}\right\} .
\end{aligned}
$$

Within the deduction made above, the output power spectral density of a multistable SR system can be obtained:

$$
\begin{aligned}
S(w) & =\int_{-\infty}^{+\infty}\langle x(t) x(t+\tau)\rangle_{\text {Average }} e^{-i w \tau} d \tau \\
& =S_{1}(w)+S_{2}(w),
\end{aligned}
$$

where $S_{1}(w)$ and $S_{2}(w)$ are the power spectral densities of the output signal and the output noise, which are derived 
from the periodic input signal and the noise, respectively, as follows:

$$
\begin{aligned}
S_{1}(w)= & \left(\frac{-c}{8 d}-\frac{1}{4} \sqrt{\left.\frac{b}{d}-\frac{c+\sqrt{c^{2}-4 b d}}{8 d}\right)}\right. \\
& \cdot \frac{\pi R_{1}^{2} \beta^{2}}{2\left(R_{0}^{2}+(2 \pi f)^{2}\right)} \delta(w-2 \pi f), \\
S_{2}(w)= & \left(\frac{c}{8 d}+\frac{1}{4} \sqrt{\frac{b}{d}}-\frac{c+\sqrt{c^{2}-4 b d}}{8 d}\right) \\
& \cdot \frac{2 R_{0}}{R_{0}^{2}+(w)^{2}} .
\end{aligned}
$$

Put $A \cos (2 \pi f t)$ as constant processing; we can get the steady state solution of the available equation (4), the potential function of $\Phi(x)$ :

$$
\Phi(x)=\frac{b}{2} x^{2}+\frac{c}{4} x^{4}+\frac{d}{6} x^{6}-A x \cos (2 \pi f t) .
$$

The probability transition rate of type 1 can be obtained:

$$
\begin{aligned}
R_{1}(t)= & \frac{\left|U^{\prime \prime}\left(-x_{1}\right) U^{\prime \prime}\left(-x_{2}\right)\right|^{1 / 2}}{2 \pi} \\
& \cdot \exp \left\{-\frac{\Phi\left(-x_{1}\right)-\Phi\left(-x_{2}\right)}{D}\right\} .
\end{aligned}
$$

Make $x_{1}=0$,

$$
\begin{aligned}
x_{0} & =-\sqrt{\frac{-1}{2 d}\left(c+\sqrt{c^{2}-4 b d}\right)}, \\
x_{2} & =\sqrt{\frac{-1}{2 d}\left(c-\sqrt{c^{2}-4 b d}\right)}-\sqrt{\frac{-1}{2 d}\left(c+\sqrt{c^{2}-4 b d}\right)}, \\
R_{0} & =\left.2 \cdot R_{1}(t)\right|_{A \cos (2 \pi f t)=0} \\
& =\frac{\sqrt{-9 b^{2}-4 b c^{2} / d+25 b^{2} / d^{2}}}{\pi} \\
\cdot & e^{-\left(-b \sqrt{c^{2}-4 b d} / 2 d+c^{2} \sqrt{c^{2}-4 b d} / 4 d^{2}+\left(-c^{2} \sqrt{c^{2}-4 b d}-c^{3}+4 b c d\right) / 3\right) / D}, \\
\frac{1}{2} R_{1} & =-\left.\frac{d R_{1}(t)}{d(A \cos (2 \pi f t))}\right|_{A \cos (2 \pi f t)=0}, \\
R_{1} \beta & =\frac{R_{0} A\left(x_{2}-x_{1}\right)}{D} .
\end{aligned}
$$

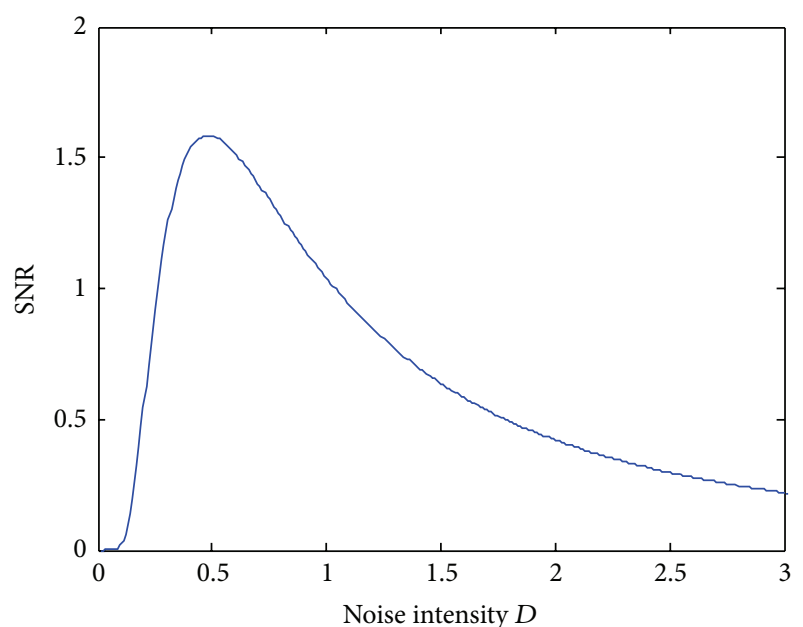

FIGURE 2: SNR versus noise intensity $D$ with $b=0.52, c=-0.31$, and $d=0.04$.

To clearly describe the energy distribution of the system output, the SNR of the system output can be calculated as follows:

$$
\begin{aligned}
\operatorname{SNR}= & \frac{\int_{0}^{\infty} S_{1}(w) d w}{S_{2}(w=2 \pi f)} \\
= & \frac{-c / 8 d-(1 / 4) \sqrt{b / d}-\left(c+\sqrt{c^{2}-4 b d}\right) / 8 d}{c / 8 d+(1 / 4) \sqrt{b / d}-\left(c+\sqrt{c^{2}-4 b d}\right) / 8 d} \\
& \cdot \frac{\pi R_{0} A^{2}(-c / d-2 \sqrt{b / d})}{4 D^{2}} .
\end{aligned}
$$

\section{The Effects of the Noise Intensity and System Parameters}

In this section, we discuss the effect of each parameter on the system SNR.

Figure 2 shows the change trends of the SNR of a multistable SR method with $b=0.52, c=-0.31$, and $d=0.04$ versus noise intensity $D$.

It can be seen from Figure 2 that the change curve of the SNR is first increased and then decreased with the variation in noise intensity $D$; therefore, there exists an optimal noise for the maximum SNR. This typical phenomenon is a signature of multistable SR. Noise plays a role in the SNR within certain range of scale.

The SNR as a function of noise intensity $D$ with different system parameters $b$ is shown in Figure 3. It is seen that the positions of the higher peaks and the lower peaks are both shifting to the left with the increase of $b$ and the SNR is decreasing with the increase of $b$.

Figure 4 shows the curves of SNR versus noise intensity $D$ with different system parameters $c$. With the increase of $c$, the whole curves are shifting to left and SNR is increasing.

Figure 5 shows the curves of SNR versus noise intensity $D$ with different system parameters $d$. With the increase of $d$, the whole curves are shifting to the left and the SNR is increasing. 


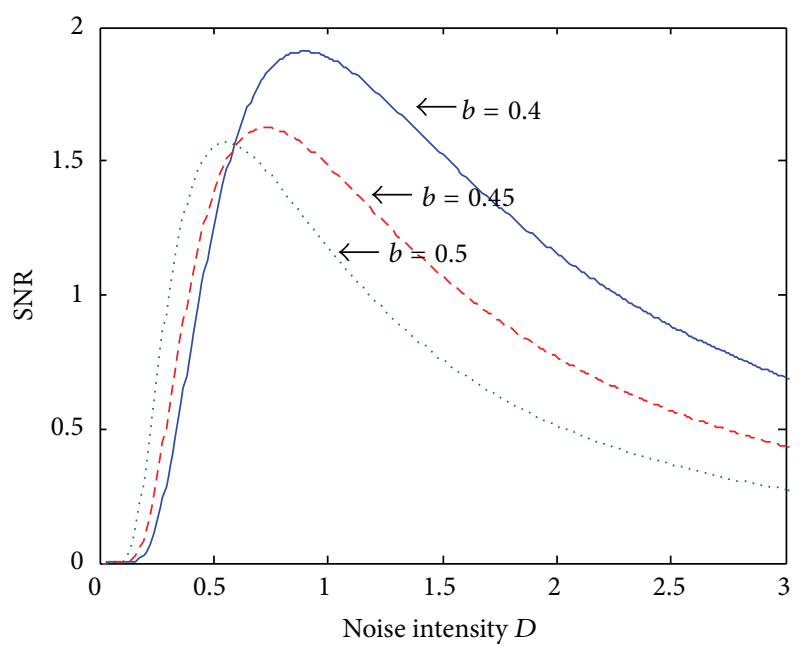

Figure 3: SNR versus noise intensity $D$ for different system parameters $b: 0.4,0.45$, and 0.5 . Other parameters are $c=-0.31$ and $d=0.04$.

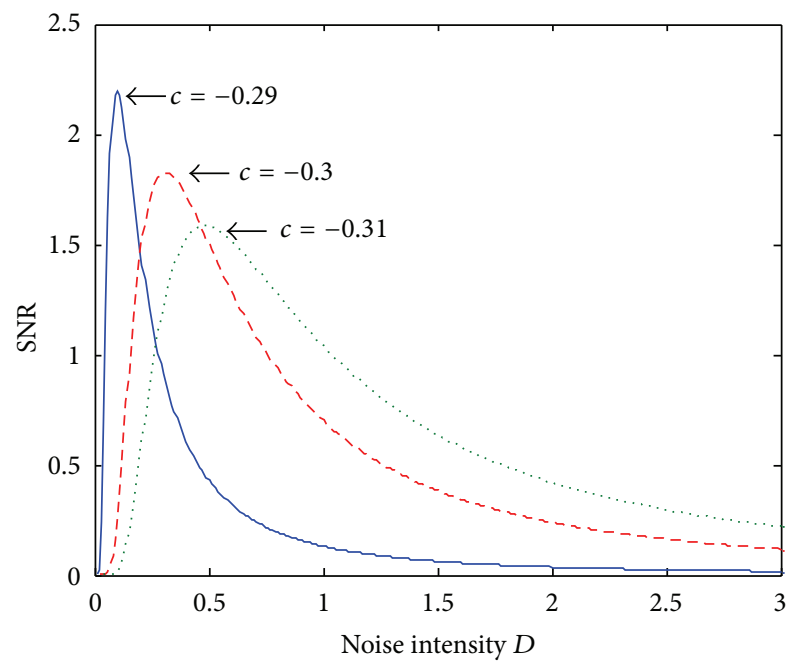

FIGURE 4: SNR versus noise intensity $D$ for different system parameters $c$ : $-0.31,-0.3$, and -0.29 . Other parameters are $b=0.52$ and $d=0.04$.

\section{The Simulation}

Take the same parameters as in Figure 2 to detect the weak signal with the multistable stochastic resonance and then let $D$ take different values; and the amplitude of the corresponding characteristic frequency is recorded; finally, the curve of amplitude versus the noise is made. It can be seen that the simulation result in Figure 6 is consistent with the analysis in Figure 2.

Take the same parameters as in Figure 3 to detect the weak signal with the multistable stochastic resonance. First, take $b$ equal to 0.4 and let $D$ take $N$ different values; then, the amplitude of the corresponding characteristic frequency is recorded and the curve of amplitude versus the noise is finally made. Second, take $b$ equal to 0.45 and 0.5 and repeat the above operation, respectively. It can be seen that the

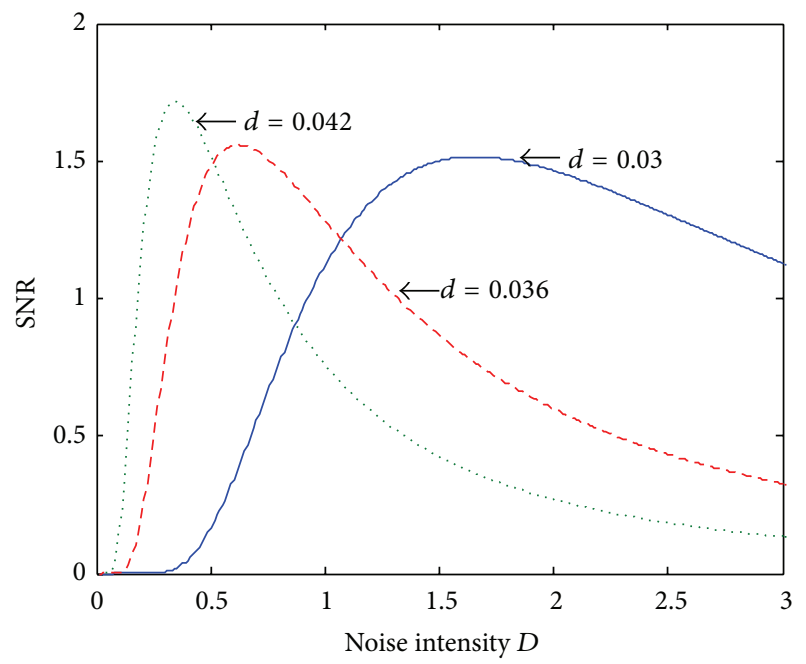

FIGURE 5: SNR versus noise intensity $D$ for different system parameters $d: 0.03,0.036$, and 0.042 . Other parameters are $b=0.52$ and $c=-0.31$.

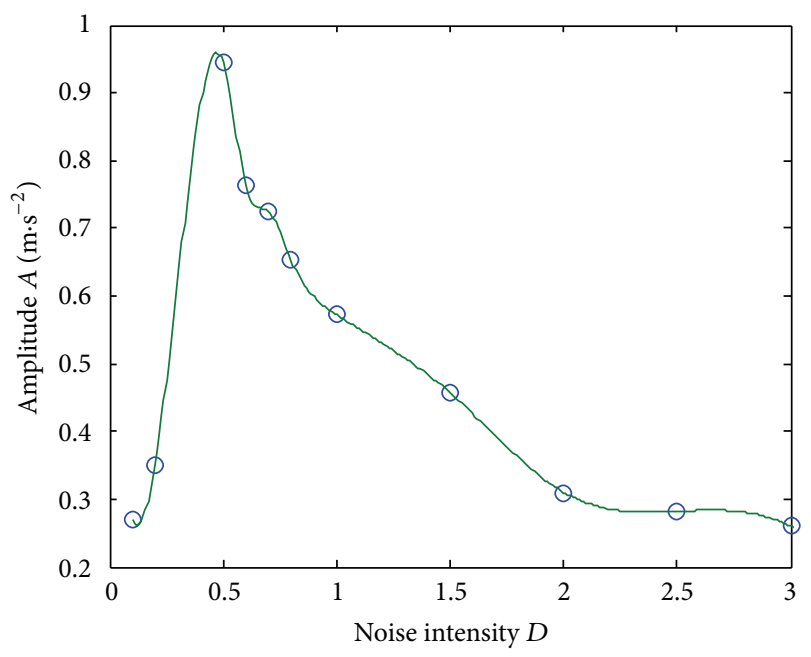

FIgURE 6: The variation curve of the output signal amplitude with the addition of noise $D$.

simulation result in Figure 7 is consistent with the analysis in Figure 3.

Take the same parameters as in Figure 4 to detect the weak signal with the multistable stochastic resonance. First, take $c$ equal to -0.31 and let $D$ take $N$ different values; then, the amplitude of the corresponding characteristic frequency is recorded and the curve of amplitude versus the noise is finally made. Second, take $c$ equal to -0.3 and -0.29 and repeat the above operation, respectively. It can be seen that the simulation result in Figure 8 is consistent with the analysis in Figure 4.

Take the same parameters as in Figure 5 to detect the weak signal with the multistable stochastic resonance. First, take $d$ equal to 0.03 and let $D$ take $N$ different values; then, the amplitude of the corresponding characteristic frequency is recorded and the curve of amplitude versus the noise is 


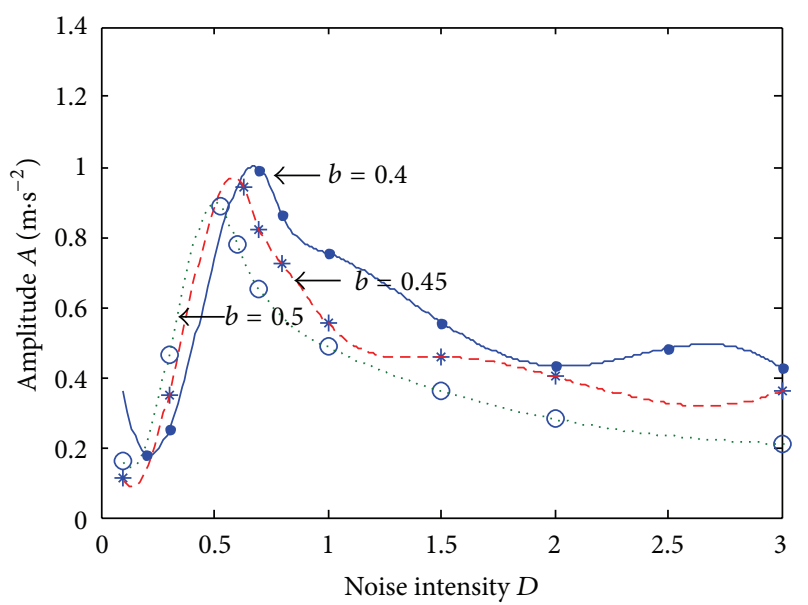

FIGURE 7: The variation curve of the output signal amplitude with the addition of noise $D$ under different $b$ value.

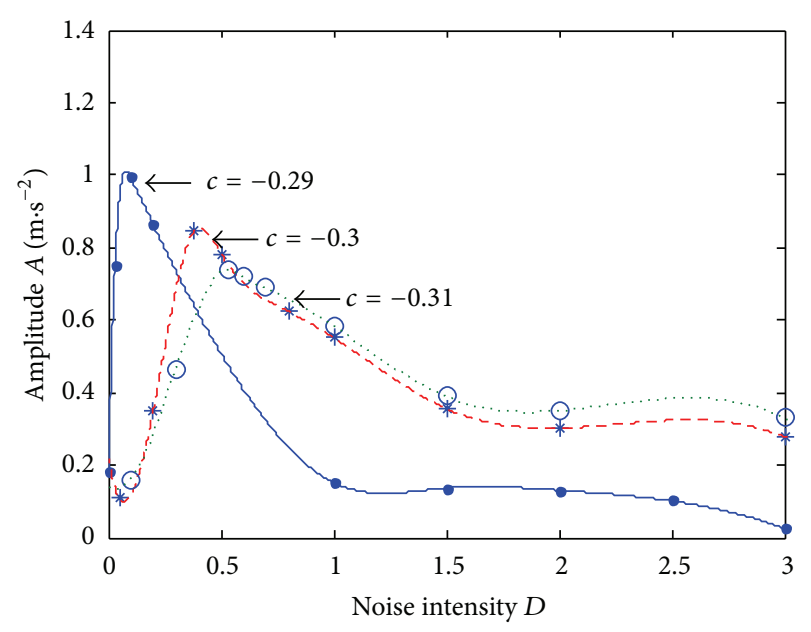

FIGURE 8: The variation curve of the output signal amplitude with the addition of noise $D$ under different $c$ value.

finally made. Second, take $d$ equal to 0.036 and 0.042 and repeat the above operation, respectively. It can be seen that the simulation result in Figure 9 is consistent with the analysis in Figure 5.

\section{Conclusion}

In the paper, we first derive the expression of the multistable system SNR. Through the research about the effects of Gauss noise and system parameters on the multistable system SNR, we can draw the following conclusions: (1) the SNR expression is applicable to arbitrary signal amplitude; (2) the curve of the SNR versus noise intensity is nonmonotonic, which is a typical phenomenon of multistable SR; (3) the SNR peak is increasing gradually with the increase of system parameters $c$ and $d$, but it is decreasing with the increase of system parameters $b$. The SNR as a function of system parameters $b, c$, and $d$ will not be described in this paper.

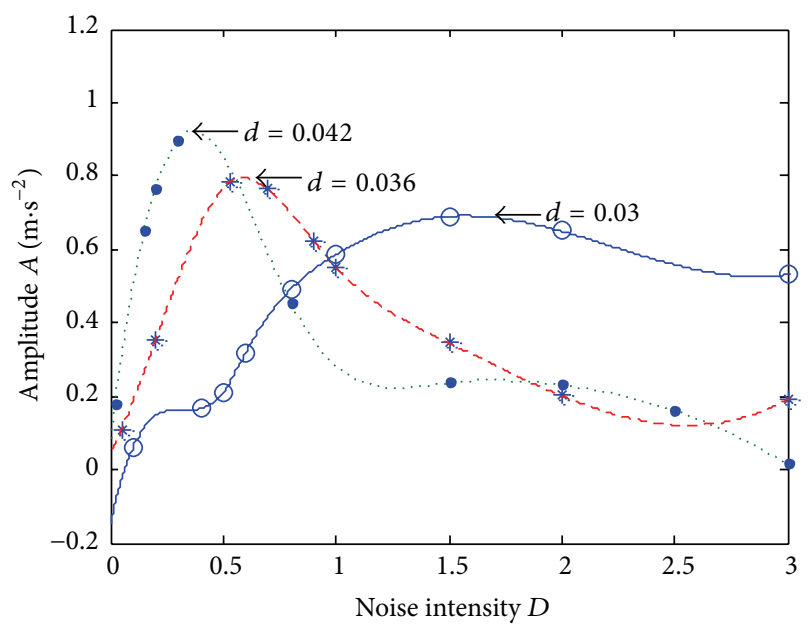

FIGURE 9: The variation curve of the output signal amplitude with the addition of noise $D$ under different $d$ value.

\section{Conflict of Interests}

The authors declare that there is no conflict of interests regarding the publication of this paper.

\section{Acknowledgments}

This work was supported by the National Natural Science Foundation of China (Grant no. 51475407), Hebei Provincial Natural Science Foundation of China (no. E2015203190), Key project of Natural Science Research in Colleges and Universities of Hebei Province of China (Grant no. ZD2015050), and the Education Department of Hebei Province Outstanding Youth Fund of China (Grant no. YQ2013020).

\section{References}

[1] R. Benzi, A. Sutera, and A. Vulpiani, "The mechanism of stochastic resonance," Journal of Physics A: Mathematical and General, vol. 14, no. 11, pp. L453-L457, 1981.

[2] R. Benzi, G. Parisi, A. Sutera, and A. Vulpiani, "Stochastic resonance in climatic change," Tellus, vol. 34, pp. 10-16, 1982.

[3] M. Yao, W. Xu, and L. Ning, "Stochastic resonance in a bias monostable system driven by a periodic rectangular signal and uncorrelated noises," Nonlinear Dynamics, vol. 67, no. 1, pp. 329333, 2012.

[4] Y. Jia, S.-N. Yu, and J.-R. Li, "Stochastic resonance in a bistable system subject to multiplicative and additive noise," Physical Review E, vol. 62, no. 2, pp. 1869-1878, 2000.

[5] J. C. Comte and S. Morfu, "Stochastic resonance: another way to retrieve subthreshold digital data," Physics Letters. A, vol. 309, no. 1-2, pp. 39-43, 2003.

[6] S. L. Lu, Q. B. He, and F. R. Kong, "Effects of underdamped step-varying second-order stochastic resonance for weak signal detection," Digital Signal Processing, vol. 36, pp. 93-103, 2015.

[7] B. McNamara and K. Wiesenfeld, "Theory of stochastic resonance," Physical Review A, vol. 39, no. 9, pp. 4854-4869, 1989.

[8] L. Gammaitoni, F. Marchesoni, and S. Santucci, "Stochastic resonance as a bona fide resonance," Physical Review Letters, vol. 74, no. 7, pp. 1052-1055, 1995. 
[9] E. Lutz, "Fractional Langevin equation," Physical Review E, vol. 64, no. 5, Article ID 051106, 2001.

[10] V. M. Gandhimathi, S. Rajasekar, and J. Kurths, "Vibrational and stochastic resonances in two coupled overdamped anharmonic oscillators," Physics Letters A, vol. 360, no. 2, pp. 279-286, 2006.

[11] R. Mankin, K. Laas, T. Laas, and E. Reiter, "Stochastic multiresonance and correlation-time-controlled stability for a harmonic oscillator with fluctuating frequency," Physical Review E, vol. 78, no. 3, Article ID 031120, 2008.

[12] S. Saikia, "The role of damping on Stochastic Resonance in a periodic potential," Physica A: Statistical Mechanics and Its Applications, vol. 416, pp. 411-420, 2014.

[13] W. Zhang and G. H. Di, "Stochastic resonance in a harmonic oscillator with damping trichotomous noise," Nonlinear Dynamics, vol. 77, no. 4, pp. 1589-1595, 2014.

[14] T. Noguchi and H. Torikai, "Ghost stochastic resonance from an asynchronous cellular automaton neuron model," IEEE Transactions on Circuits and Systems II: Express Briefs, vol. 60, no. 2, pp. 111-115, 2013.

[15] I. G. Silva, O. A. Rosso, M. V. Vermelho, and M. L. Lyra, "Ghost stochastic resonance induced by a power-law distributed noise in the FitzHugh-Nagumo neuron model," Communications in Nonlinear Science and Numerical Simulation, vol. 22, no. 1-3, pp. 641-649, 2015.

[16] Y. Xu, Y. Li, J. Li, J. Feng, and H. Zhang, "The phase transition in a bistable Duffing system driven by Lévy noise," Journal of Statistical Physics, vol. 158, no. 1, pp. 120-131, 2015.

[17] M. J. He, W. Xu, and Z. K. Sun, "Dynamical complexity and stochastic resonance in a bistable system with time delay," Nonlinear Dynamics, vol. 79, no. 3, pp. 1787-1795, 2015.

[18] R.-L. Lang, L. Yang, H.-L. Qin, and G.-H. Di, “Trichotomous noise induced stochastic resonance in a linear system," Nonlinear Dynamics, vol. 69, no. 3, pp. 1423-1427, 2012.

[19] J. F. Lindner, B. J. Breen, M. E. Wills, A. R. Bulsara, and W. L. Ditto, "Monostable array-enhanced stochastic resonance," Physical Review E, vol. 63, no. 5, Article ID 051107, 2001.

[20] M. S. Siewe, F. M. M. Kakmeni, and C. Tchawoua, "Resonant oscillation and homoclinic bifurcation in a $\phi^{6}$-Van der Pol oscillator," Chaos, Solitons \& Fractals, vol. 21, no. 4, pp. 841-853, 2004.

[21] J. Li, X. Chen, and Z. He, "Multi-stable stochastic resonance and its application research on mechanical fault diagnosis," Journal of Sound and Vibration, vol. 332, no. 22, pp. 5999-6015, 2013.

[22] D. Han, P. li, S. An, and P. Shi, "Multi-frequency weak signal detection based on wavelet transform and parameter compensation band-pass multi-stable stochastic resonance," Mechanical Systems and Signal Processing, vol. 70-71, pp. 995-1010, 2016.

[23] L. B. Kish, S. Khatri, and S. Sethuraman, "Noise-based logic hyperspace with the superposition of $\mathrm{N}^{2}$ states in a single wire," Physics Letters A, vol. 373, no. 22, pp. 1928-1934, 2009.

[24] I. M. Sokolov, W. Ebeling, and B. Dybiec, "Harmonic oscillator under Lévy noise: unexpected properties in the phase space," Physical Review E, vol. 83, no. 4, Article ID 041118, 2011.

[25] P. Jung and F. Marchesoni, "Energetics of stochastic resonance," Chaos, vol. 21, no. 4, Article ID 047516, 2011.

[26] S. Fauve and F. Heslot, "Stochastic resonance in a bistable system," Physics Letters A, vol. 97, no. 1-2, pp. 5-7, 1983.

[27] L. F. Lafuerza, P. Colet, and R. Toral, "Nonuniversal results induced by diversity distribution in coupled excitable systems," Physical Review Letters, vol. 105, no. 8, Article ID 084101, 2010. 


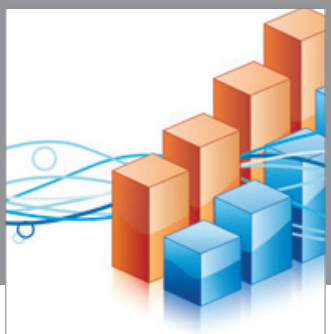

Advances in

Operations Research

vatem alat4

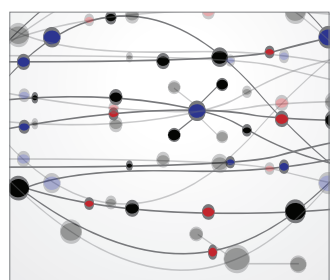

\section{The Scientific} World Journal
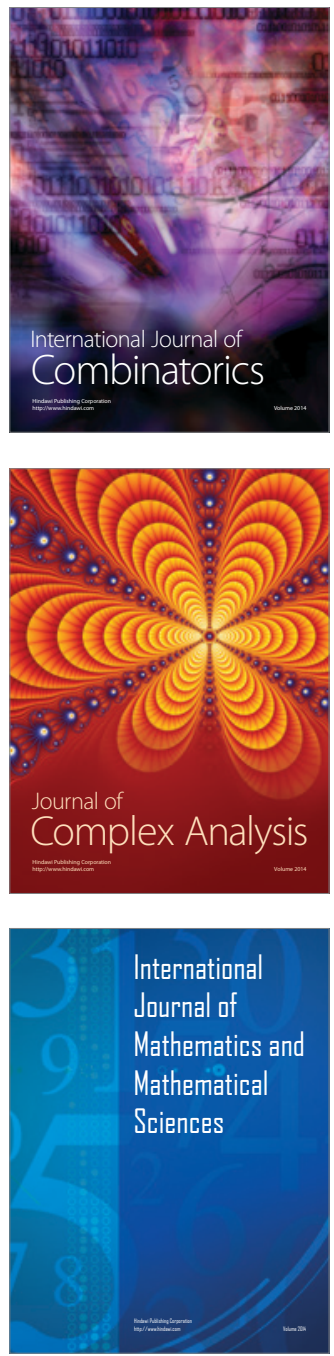
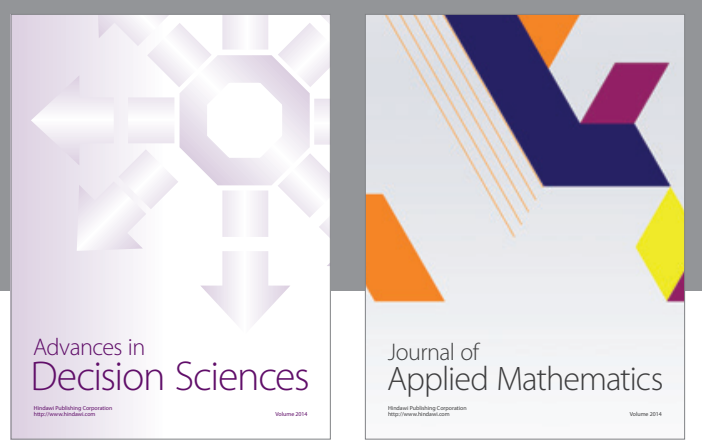

Algebra

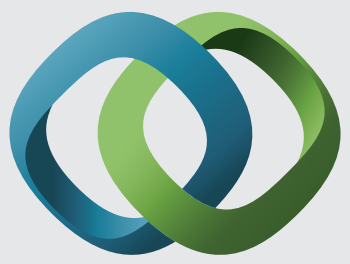

\section{Hindawi}

Submit your manuscripts at

http://www.hindawi.com
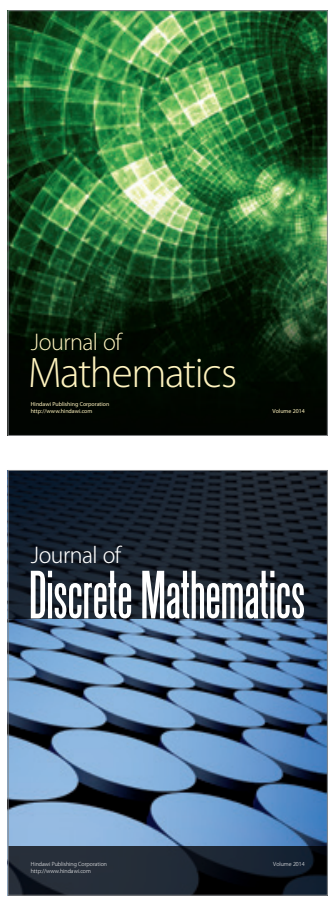

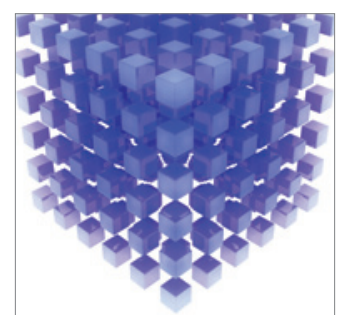

Mathematical Problems in Engineering
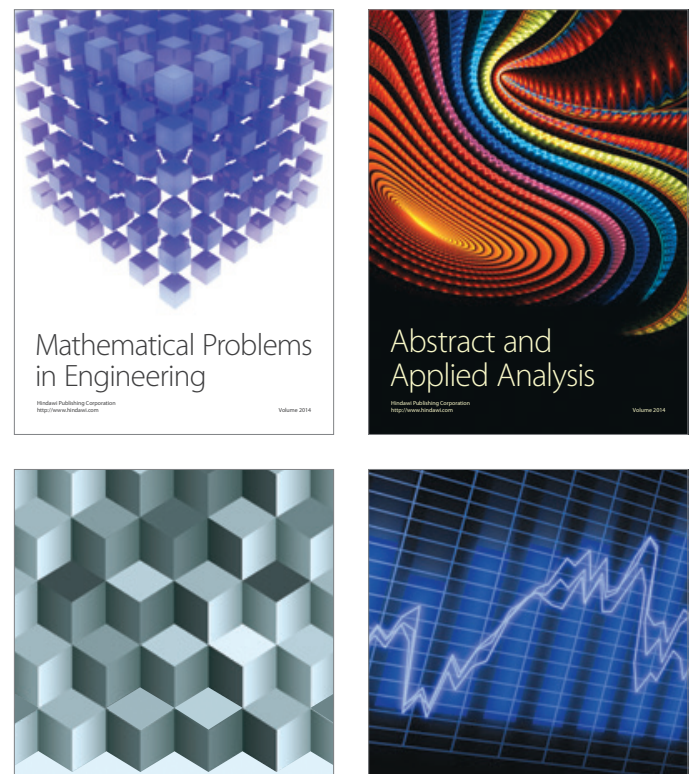

Journal of

Function Spaces

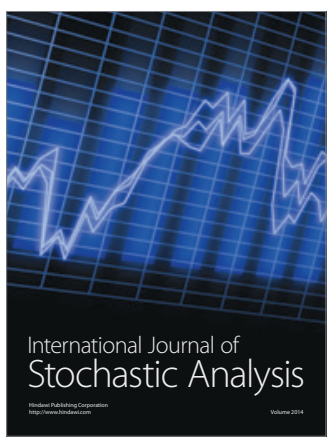

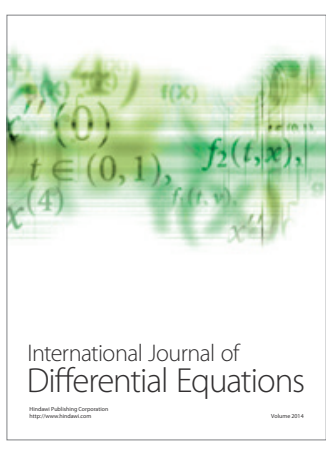
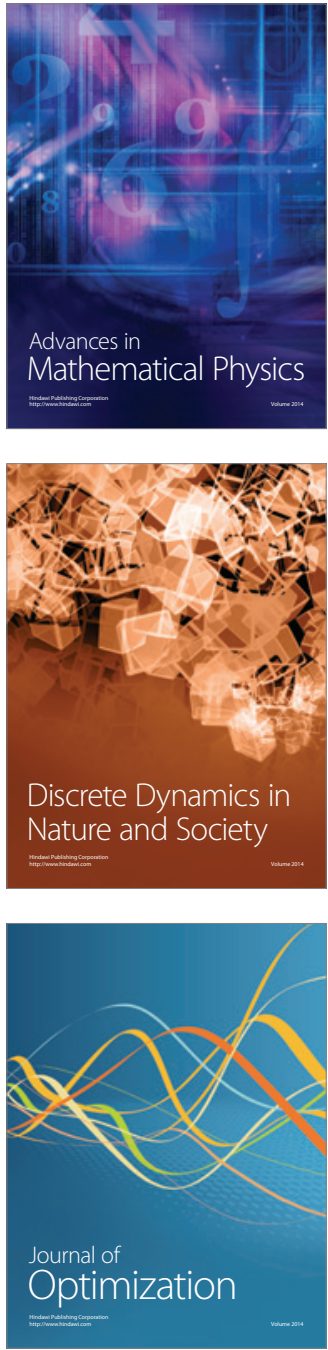